REGARDS

SUR LEECONOMIE ALLEMANDE

BULLETIN ECONOMIQUE DU CRAC

\section{Regards sur l'économie allemande}

Bulletin économique du CIRAC

$95 \mid 2010$

Varia

\title{
Le secteur de la santé face au droit de la concurrence
}

\section{Géraldine Demme}

\section{(2) OpenEdition}

\section{Journals}

Édition électronique

URL : http://journals.openedition.org/rea/4032

DOI : $10.4000 /$ rea. 4032

ISBN : 978-2-8218-0886-7

ISSN : 1965-0787

\section{Éditeur}

CIRAC

\section{Édition imprimée}

Date de publication : 1 mars 2010

Pagination : 27-32

ISSN : 1156-8992

\section{Référence électronique}

Géraldine Demme, "Le secteur de la santé face au droit de la concurrence », Regards sur l'économie allemande [En ligne], 95 | mars 2010, mis en ligne le 01 mars 2012, consulté le 01 mai 2019. URL :

http://journals.openedition.org/rea/4032 ; DOI : 10.4000/rea.4032 


\title{
Le secteur de la santé face au droit de la concurrence
}

\author{
Géraldine Demme
}

Le malade est-il un client? La santé est-elle un bien de consommation comme les autres? Si la réponse à ces questions est négative, les rapports entre le droit de la concurrence et le marché de la santé sont alors nécessairement plus complexes que dans d'autres secteurs de l'économie. Indissolublement lié à un système d'économie de marché, fondé sur les forces spontanées des agents économiques qui y interviennent, le droit de la concurrence est d'inspiration libérale. Jusqu'à quel point ces règles sont-elles compatibles avec le principe de solidarité auquel le secteur de la santé fait appel?

A travers ses réformes du système d'assurance maladie, dont la dernière en date (création du "Fonds pour la Santé »: Gesundheitsfonds) est entrée en vigueur le 01-01-2009, I'Allemagne cherche à donner une réponse équilibrée, entre respect des impératifs de politique sociale et maintien d'une concurrence efficace sur le marché de la santé. Toutefois, le législateur se borne bien souvent à injecter des doses très limitées de concurrence - notamment celles grâce auxquelles il espère une baisse de la dépense publique en matière de santé - sans pour autant s'en remettre résolument aux mécanismes du marché dans ce domaine.

Or, concurrence et santé publique ne sont pas antinomiques, bien au contraire, leur association consolide l'intérêt général à long terme. Le gardien de la concurrence qu'est l'Office fédéral des Cartels (Bundeskartellamt), plaide ainsi depuis longtemps pour un renforcement de la concurrence dans le domaine de la santé, considérant qu'elle génère les conditions les plus favorables pour le respect du principe de solidarité. Et il ne manque pas d'infliger des amendes importantes aux entreprises qui transgressent les règles de la concurrence, là où en a laissé la possibilité un législateur qui n’a pas voulu trancher entre étatisation et marché.

\section{L'influence des spécificités du marché de la santé sur le droit de la concurrence}

La rivalité entre fournisseurs de biens et prestataires de services en vue d'obtenir la solution la plus satisfaisante possible est l'élément capital sur lequel se fonde notre organisation économique. En effet, la libre concurrence entraîne la réduction des prix et la hausse de la qualité des biens et services. Dans un tel système, l'Etat n'intervient, en principe, que pour garantir le libre jeu de la concurrence entre les différents acteurs sur le marché. Si ce constat est commun et vaut pour tous les secteurs de l'économie, y compris celui de la santé, il nécessite toutefois des précisions dans ce domaine bien spécifique.

La santé est un secteur clé de l'économie allemande. Selon les données de Destatis, il représente 4,4 millions d'emplois : une personne sur dix travaille dans ce domaine en Allemagne. Les dépenses annuelles pour la santé se chiffrent à 252,8 milliards $€$ en 2007 (à savoir $3070 €$ en moyenne par tête), ce qui équivaut à $10,4 \%$ du PIB. L'importance de ce secteur va croissant. Les dépenses sont en hausse constante : de 2005 à 2007, elles ont augmenté d'environ 13 milliards $€$. Cette tendance n'est pas près de s'inverser, pour plusieurs raisons. Tout d'abord, les dépenses de santé s'accroissent corrélativement à l'amélioration du niveau de vie. En outre, les avancées médico-techniques permettent la création de nouvelles méthodes de soins plus performantes mais aussi plus onéreuses. Enfin, les mutations démographiques jouent un rôle essentiel : nous vivons plus longtemps et demandons davantage de soins.

Importance économique croissante du secteur de la santé 
Les spécificités du marché de la santé justice distributive et solidarité

Un haut degré de régulation nationale des systèmes d'assurance maladie

Mais les entreprises actives sur ce secteur restent soumises au droit de la concurrence, national et européen

L'entrave aux importations parallèles de médicaments par les entreprises pharmaceutiques
Si, par son importance, le marché de la santé a une incidence certaine sur la vie économique en Allemagne, il agit, par sa fonction, sur la vie - et la survie - des citoyens. Ainsi, ce marché n'est pas uniquement déterminé par les besoins du libre échange de biens et prestations, mais également par des considérations de politique sociale. Nul besoin de mentionner que dans un Etatsocial tel que l'Allemagne (art. 20, alinéa 1er de la Loi fondamentale), l'accès aux soins et leur qualité ne doivent pas dépendre du portefeuille du malade. C'est dans ce sens que le gouvernement allemand constatait en 2006 que le malade n'est pas un client et que la santé n'est pas un bien de consommation, mais un bien existentiel de chacun. De ce fait, le marché de la santé montre des spécificités qui l'éloignent d'autres marchés de biens et de prestations de services.

En effet, la protection de la santé est d'intérêt général et repose sur des exigences de justice distributive. Cela ne signifie toutefois pas qu'une telle approche soit étrangère au droit de la concurrence. Ainsi, il intègre ce principe par exemple en matière des ententes où, pour qu'un accord anticoncurrentiel entre entreprises qui produit des gains d'efficacité échappe à l'interdiction, les gains obtenus doivent être répercutés au moins en partie sur les utilisateurs. C'est par exemple le cas si une coopération entre deux producteurs de freins à main pour bicyclettes permet plus rapidement la mise sur le marché de produits plus sûrs, ou si d'une telle synergie résulte la réduction des coûts par unité produite qui est partiellement transmise aux consommateurs sous la forme d'une baisse des prix finaux. Mais le secteur de la santé demande davantage, à savoir une redistribution entre citoyens : tous doivent avoir accès aux soins, y compris les plus faibles économiquement ; c'est le principe de solidarité qui prime.

Ces considérations de politique sociale sont la raison pour laquelle le domaine de la santé se caractérise par une forte intervention régulatrice. Ainsi, les prix des médicaments sont soumis au contrôle de l'Etat: la Arzneimittelpreisverordnung (AMPreisV, règlement concernant les prix des médicaments) qui prévoit les marges des grossistes pharmaceutiques et des pharmacies pour les médicaments délivrés uniquement sur ordonnance (ce qui rend impossible la concurrence par les prix de ces produits entre pharmacies), ou les montants maxima pris en charge par les systèmes nationaux d'assurance maladie (Erstattungshöchstbeträge der Krankenversicherungsorganisationen, cf. § 31 al. 2a SBG V, Sozialgesetzbuch), en constituent autant d'exemples en Allemagne.

Contrairement à d'autres domaines, les dispositifs nationaux sont moins influencés par le droit communautaire ; la souveraineté des Etats en la matière est très étendue. C'est ainsi que l'art. 168, § 5 du Traité sur le fonctionnement de l'Union européenne (TFUE) exclut toute harmonisation communautaire des dispositions législatives et réglementaires des Etats membres concernant la santé publique. La santé publique est tout d'abord affaire des Etats membres. Ceci n'empêche pas que les entreprises actives sur le marché de la santé de l'UE sont soumises tant aux règles nationales qu'européennes du droit de la concurrence.

Etant donné que le prix de revente des médicaments est régulé de façon autonome par chaque Etat membre et que le niveau de vie diffère entre les pays, le prix des mêmes produits fluctue à l'intérieur de l'UE. Ceci pose bien souvent le délicat problème des importations parallèles de médicaments, plus précisément celui de la licéité ou non de leur limitation par les entreprises pharmaceutiques. Par « importation parallèle », on entend le fait d'importer, puis de distribuer un produit d'un État membre dans un autre Etat membre de I'Union européenne, en dehors - c'est-àdire en parallèle - du réseau de distribution mis en place par le fabricant ou son distributeur agréé. L'importateur parallèle cherche ainsi à profiter d'un différentiel de prix existant entre les pays, pour accroître sa marge bénéficiaire.

L'attitude des autorités et tribunaux européens est, en principe, favorable aux importations parallèles. Ils cherchent même à favoriser cette pratique afin d'harmoniser les prix à l'intérieur du marché commun. Selon eux, tout accord entre producteur et distributeur qui tend à reconstituer les cloisonnements nationaux dans le commerce entre Etats membres est susceptible de contrarier l'objectif de l'intégration du marché unique. C'est à de maintes reprises que la Cour de justice de I'Union européenne (CJUE, anciennement Cour de justice des Communautés euro- 
péennes) a qualifié des accords visant à rendre plus difficile l'interpénétration des marchés nationaux - notamment ceux visant à interdire ou à restreindre les importations parallèles - d'accords restrictifs de concurrence, tout en condamnant les entreprises à des amendes conséquentes, par exemple 90 millions $€$ pour Volkswagen en 2003 (aff. $n^{\circ} \mathrm{C}-338 / 00$ ).

La solution peut cependant varier lorsque sont en cause des médicaments. Le 6 octobre dernier, la Cour a rendu une décision concernant la possibilité d'un laboratoire pharmaceutique d'entraver le commerce parallèle (aff. jointes $n^{\circ} C-501 / 06 P$, C-513/06 P, C-515/06 P et C-519/06 P). Les faits à l'origine de cet arrêt sont les suivants : GlaxoSmithKline (GSK), fabricant de produits pharmaceutiques, a entrepris de limiter l'importation parallèle de ses médicaments, en convenant avec des grossistes des prix différents pour certains médicaments selon que ces grossistes les revendaient à l'intérieur du pays ou les exportaient. La Commission européenne avait retenu l'existence d'une restriction de la concurrence prohibée par l'art. 81 \$ 1 du Traité CE (devenu l'art. $101 \S 1$ TFUE) et avait refusé toute exemption individuelle de cette pratique au titre de l'art. 81 § 3 du Traité CE (art. 101 § 3 TFUE).

Cette décision a été partiellement annulée par le Tribunal de première instance de l'Union européenne par un arrêt du 27 septembre 2006. Bien que les juges aient confirmé que l'accord entre GSK et ses grossistes était effectivement contraire à l'art. $81 \S 1$ du Traité CE (art. $101 \S 1$ TFUE), ils ont reproché à la Commission de ne pas avoir entrepris un examen rigoureux des conditions d'application d'une éventuelle exemption. Le tribunal souligne dans son arrêt la gravité particulière d'une telle omission " dans le cadre d'un contexte juridique et économique, tel que celui caractérisant le secteur pharmaceutique, où le jeu de la concurrence est faussé par la présence de réglementations étatiques » (point 276). En effet, si les importations parallèles peuvent, en principe, conduire à une réduction des prix dans les pays où les produits sont vendus plus cher, le constat n'est pas le même dans un secteur caractérisé par la réglementation des prix des médicaments. Le TPI souligne en outre l'importance de la concurrence par l'innovation dans le secteur pharmaceutique où la $\mathrm{R} \& \mathrm{D}$ est coûteuse et risquée. Selon les juges, la « différenciation des prix permet alors [aux laboratoires pharmaceutiques] de récupérer le coût de $R \& D$ auprès des consommateurs finals qui sont prêts à le payer » (point 271). Dans son récent arrêt, la Cour de justice vient de confirmer la décision du Tribunal de première instance, en mettant en avant que la Commission avait eu tort de ne pas "prendre en compte les caractères et les éventuelles spécificités du secteur concerné par l'accord (...), dont notamment les spécificités structurelles du secteur pharmaceutiques en cause » (points 103, 104). La Commission devra donc rendre à nouveau une décision en incluant ces spécificités.

Eu égard aux impératifs particuliers auxquels il est soumis, le marché de la santé fait l'objet d'une plus grande intervention étatique de nature à modifier ou empêcher le jeu normal de la concurrence. Les autorités et tribunaux chargés de veiller à la préservation de la concurrence doivent prendre en compte ces particularités dans leurs analyses contextuelles.

\section{Le domaine d'influence du droit de la concurrence sur le marché de la santé}

La liberté de la concurrence et l'intérêt général de tous à la qualité du système de santé ne sont pas, loin s'en faut, antinomiques. La concurrence - décrite comme " méthode de découverte » (Entdeckungsverfahren) par F.A. von Hayek (Prix Nobel d'économie en 1974) - permet de coordonner de façon flexible les efforts privés en vue d'obtenir leur meilleur usage possible. Sous peine de ne pas pouvoir subsister sur le marché, les opérateurs économiques soumis à une pression concurrentielle doivent adapter continuellement leurs activités aux attentes et nécessités des clients. Ils agissent ainsi naturellement, non seulement dans leur propre intérêt, mais également dans celui du consommateur. Ce dernier apparaît comme le souverain d'un tel processus économique sélectif. La concurrence permet ainsi une meilleure allocation des ressources, une réduction des prix et une augmentation de la qualité des biens et services. Moteur du libre échange sur le marché, elle stimule également la $R \& D$, en d'autres termes, elle augmente le progrès technique. Au vu
Les autorités et tribunaux doivent prendre en compte les spécificités du secteur pharmaceutique
Absence d'antinomie entre le droit de la concurrence et le secteur de la santé 
Les réformes en vue de renforcer la concurrence...

... comportent des éléments d'économie administrée

II s'ensuit un manque de concurrence entre les caisses d'assurance maladie

L'annonce commune de la levée de primes d'assurance complémentaires... de ses effets bénéfiques, les pouvoirs publics cherchent à consolider un niveau efficace de concurrence sur le marché, que ce soit par le contrôle des comportements des entreprises ou par la surveillance de la structure du marché.

Renforcer la concurrence sur le marché, c'est également le souhait du législateur allemand dans le secteur de la santé. La Loi de renforcement de la concurrence dans l'assurance maladie légale (GKV-Wettbewerbsstärkungsgesetz, GKV-WSG) de 2007 affirme cet objectif jusque dans son intitulé. Mais contrairement à ce que laisse entendre celui-ci, cette loi a mis également en place des éléments d'économie administrée (Hassenteufel, 2006). Ceci est notamment le cas avec la création d'un « Fonds pour la santé » (Gesundheitsfonds). Ce fonds, organisé de façon centralisée par l'Etat, se compose de cotisations et de ressources fiscales. Le budget est, par la suite, réparti entre les caisses.

Le Gesundheitsfonds est critiquable et critiqué sous l'angle du droit de la concurrence car il a mené à un taux de cotisation unique et déterminé par l'État des caisses affiliées au régime légal (voir REA 77/06). En encadrant le budget des caisses et en les privant de leur autonomie dans la fixation des cotisations, le législateur a éliminé un élément crucial à l'exercice de la concurrence entre les différentes caisses d'assurance maladie: la concurrence par les prix. Cela a amené Bernhard Heitzer, président du Bundeskartellamt jusqu'en 2009, à condamner à plusieurs reprises le renforcement du rôle régulateur de l'Etat qui en résulte. Selon lui, le Fonds pour la santé, instrument d'une économie planifiée, n'est pas capable de produire un effet positif équivalent à celui obtenu par une concurrence libre par les prix. Une perte du lien entre demande, coûts et prestations en résulte.

Le paramètre de la concurrence par les cotisations n'est toutefois pas totalement exclu par le Fonds pour la santé. Le système est en effet censé rendre plus efficace le travail gestionnaire des caisses : celles-ci peuvent demander un montant complémentaire à leurs assurés si le financement provenant du Fonds pour la santé ne leur suffit pas. Ce versement consiste en une prime d'assurance supplémentaire, soit sous forme d'une somme forfaitaire pouvant aller jusqu'à $8 €$ mensuels par assuré, soit par un montant pouvant s'élever jusqu'à $1 \%$ du revenu brut de chaque assuré et plafonné à $37,50 €$ par mois ; à l'inverse, elles peuvent faire bénéficier leurs assurés de ristournes si leur gestion génère un surplus. II est pourtant notoire que la marge de manœuvre laissée aux caisses est dorénavant fort réduite et n'équivaut pas à une libre concurrence dans la détermination des cotisations ellesmêmes. II ne fait pas de doute non plus qu'une concurrence dans la qualité des services n'est pas à même de combler l'absence de concurrence par les prix en matière de santé : la bonne perception de la qualité des prestations n'est pas chose facile dans ce domaine car, dans la plupart des cas, le patient en est simplement incapable, tant au plan de la connaissance du produit que par son état émotionnel.

Le fait que plusieurs caisses d'assurance, dont la Deutsche Angestellten-Krankenkasse (DAK), la troisième plus grande caisse d'Allemagne, ont annoncé en janvier de cette année - un an seulement après l'instauration du Fonds pour la santé qu'elles vont lever des primes d'assurances complémentaires de $8 €$, confirme une fois de plus que des interventions directes de l'Etat risquent de mener à des inefficacités dans l'allocation des ressources, pénalisant in fine les consommateurs. Les caisses concernées justifient cette pratique par la nécessité de combler le manque de financement obtenu par le budget issu du Fonds. Plus récemment encore, deux autres caisses, la BKK Heilberufe et la GBK Köln, ont fait, elles aussi, part de la mise en place d'une prime supplémentaire. Contrairement aux caisses précédemment mentionnées qui demandent une prime forfaitaire fixe, ces organismes appliqueront une somme proportionnelle au revenu de leurs assurés qui peut s'élever jusqu'au plafond légal de 37,50 € par mois. D'autres organismes suivront.

L'annonce conjointe faite par plusieurs caisses d'assurance de la levée d'un montant complémentaire et identique de $8 €$ par assuré est également source d'inquiétudes du point de vue de la concurrence. Un tel comportement peut constituer une entente anticoncurrentielle au sens du $\S 1$ de la loi allemande contre les entraves à la concurrence (Gesetz gegen Wettbewerbsbeschränkungen, GWB), si les caisses d'assurances sont qualifiées d'entreprises au sens de cette loi. Contrairement à la jurisprudence communautaire qui écarte souvent l'activité économique des orga- 
nismes chargés de la gestion de régimes légaux d'assurance maladie en raison de leur caractère social (par exemple CJCE, 16 mars 2004, AOK, C-264/01, C-306/01, C-354/01 et C-355/01), en Allemagne, les caisses d'assurance maladie sont, en principe, considérées par la jurisprudence comme étant des entreprises au sens de la loi GWB. Le système juridique allemand fait néanmoins bénéficier ces organismes d'une position très singulière en la matière. Ainsi, le § 69 SBG V (Sozialgesetzbuch) rend les règles de la loi GWB en partie inapplicables aux rapports entre les caisses d'assurance et leurs prestataires de services, tels que les médecins, les hôpitaux ou les entreprises pharmaceutiques. Par le biais des règles du $S G B$, qui remplacent les dispositions écartées de la loi $G W B$, le législateur a souhaité renforcer le poids des caisses d'assurance dans leurs négociations. Ainsi, elles peuvent grouper leurs achats de médicaments au-delà même de ce que le droit de la concurrence considère comme étant raisonnable d'un point de vue du pouvoir de la demande (pour un avis critique des effets qui en résultent à long terme, voir Bundeskartellamt, rapport d'activité 2005/2006, p. 85). Cependant, cette particularité ne les fait pas échapper à l'interdiction des ententes anticoncurrentielles au cas où elles s'accordent sur les prix qu'elles demandent aux assurés.

Les caisses d'assurance concernées se défendent de toute entente anticoncurrentielle. C'est ainsi que Florian Lanz, porte-parole de l'association centrale des assurances maladie affiliées au régime légal (Spitzenverband der Gesetzlichen Krankenversicherungen), interprète cette hausse comme étant simplement la suite logique de l'instauration du Fonds pour la santé : la levée du montant de $8 €$ ne signifie, selon lui, rien d'autre que le recours par ces caisses au montant maximum de la prime forfaitaire prévue par la loi en cas de manque de financement. Toutefois, un tel comportement collectif de différentes caisses d'assurance ne peut pas être considéré comme a priori irréprochable du point de vue de la concurrence. En effet, avec l'instauration de la cotisation unique, le seul paramètre de concurrence par les prix laissé par le législateur aux caisses est celui des primes et des ristournes. Si les caisses s'accordent sur la levée simultanée de primes supplémentaires, le citoyen en pâtira. II est donc essentiel que les autorités de contrôle s'assurent du bon déroulement du jeu de concurrence, là où le législateur en a laissé la possibilité. Ceci est également l'avis de l'Office fédéral des Cartels qui, suite à plusieurs plaintes, examine actuellement le dossier.

Et ce n'est pas l'unique préoccupation des gardiens de la concurrence en matière de santé : ces derniers temps, nous assistons à une augmentation de leurs activités dans ce domaine. En effet, les producteurs de médicaments, de dispositifs médicaux et de leurs accessoires font de plus en plus l'objet de contrôles et de condamnations de la part des autorités de concurrence, tant nationales que communautaires. La Commission européenne a même ouvert en 2008 une enquête sur l'existence d'éventuelles pratiques anticoncurrentielles dans le secteur pharmaceutique. Et les amendes peuvent coûter cher aux entreprises concernées : selon l'art. 23, al. 2 du Règlement (CE) n 1/2003 du Conseil du 16 décembre 2002, la Commission européenne peut infliger aux entreprises contrevenantes une amende allant jusqu'à $10 \%$ de leur CA total réalisé au cours de l'année précédente ; le $\$ 81$ GWB octroie une possibilité équivalente au Bundeskartellamt.

Que ce dernier en fasse usage, c'est la douloureuse leçon qu'ont dû tirer récemment deux entreprises dans le secteur des dispositifs médicaux. Dans les deux affaires, des ententes sur les prix entre fabricants et distributeurs étaient en cause, et notamment la délimitation entre politique de prix restrictif et prix conseillé. La première affaire concerne le marché des aides auditives. Un audioprothésiste avait publié ses prix pour les aides auditives de différents producteurs sur Internet. Ses prix proposés pour des prothèses auditives de la marque Phonak étaient, en partie, bien en-dessous des prix communément pratiqués sur le marché. D'autres audioprothésistes se sont alors tournés vers Phonak pour se plaindre de ce comportement. Phonak a alors refusé de livrer ses produits à l'audioprothésiste qui avait adopté les tarifs plus bas, espérant ainsi l'inciter à revoir sa politique de prix. L'objectif fut atteint. Ce phénomène est également connu sous l'appellation « adverse action after distributor noise ». La seconde affaire concerne, pour sa part, le marché des lentilles de contact. En l'espèce, Ciba-Vision, producteur de lentilles de contact, surveillait les prix de revente que les distributeurs de ses produits pratiquaient sur ...est problématique du point de vue du droit des ententes

Activité de contrôle croissante sur le marché pharmaceutique, tant par la

Commission européenne...

... que par

l'Office fédéral des Cartels 
Condamnation récente de fabricants de dispositifs médicaux pour des pratiques de prix imposés
Internet. Si ces prix étaient inférieurs d'un certain montant à ceux conseillés par Ciba, l'entreprise entrait en contact avec le distributeur concerné afin de le persuader d'augmenter ses prix.

Que ce soit en droit allemand ou en droit communautaire de la concurrence, l'imposition d'un prix fixe ou minimum par le fabricant aux distributeurs indépendants est interdit, contrairement aux prix maxima ou conseillés qui sont des pratiques licites. Un prix maximum ou conseillé peut cependant équivaloir à un prix fixe ou minimal "à la suite d'une pression exercée par l'une des parties ou de mesures d'incitation prises par elle » (art. 4 Règlement CE n²790/1999), et notamment à travers « des sanctions, des retards ou suspensions de livraison ou la résiliation de l'accord en cas de non respect d'un niveau de prix donné » (point 47 des Lignes directrices sur les restrictions verticales). Le Bundeskartellamt a observé dans l'affaire Ciba-Vision que tout contact entre producteurs et commerçants concernant les prix est interdit selon le $\S 1 G W B$, lorsque le producteur s'intéresse concrètement au prix donné par le distributeur et que les deux s'accordent ainsi sur le comportement futur du commerçant. Le fait qu'il s'agissait de deux distributeurs visibles sur Internet a également joué un rôle important. C'est en ce sens que le gardien de la concurrence a souligné dans l'affaire Phonak que le fait d'éliminer un offreur pratiquant une politique de prix agressive sur Internet accentue la stabilité des prix déjà trop importante sur le marché des appareils auditifs. C'est pour avoir enfreint l'interdiction des prix minima imposés que le Bundeskartellamt a condamné Ciba-Vision en septembre 2009 à 11,5 millions $€$ d'amende, ainsi que Phonak en octobre 2009 à 4,2 millions $€$.

LE SECTEUR DE LA SANTÉ FAIT L'OBJET D'UN INTÉRÊT GRANDISSANT de la part des autorités de la concurrence, tant nationales que communautaires, et on ne peut que les en féliciter. En effet, la contradiction supposée entre droit social et principes de concurrence ne se vérifie souvent pas dans les faits, bien au contraire. Les tribunaux et autorités de la concurrence cherchent à consolider une réelle compétition entre les opérateurs sur le marché. Cette compétitivité est vertueuse car, soumises à une telle rivalité, les entreprises doivent redoubler leurs efforts pour se maintenir, que ce soit en baissant leurs coûts ou en augmentant la qualité de leurs produits ou services. In fine, ce processus économique profite au citoyen. Ceci est également vrai pour le marché de la santé, et c'est la raison pour laquelle la plus grande satisfaction des objectifs d'une politique sociale passe en principe par le maintien d'un niveau de concurrence efficace avec une intervention mesurée de l'Etat. Au lieu de créer davantage d'immunités au profit des acteurs du marché de la santé, un contrôle par les autorités de la concurrence est nécessaire pour favoriser en ce domaine le progrès à moindre prix en faveur de l'intérêt général à long terme.

\section{Indications bibliographiques}

- BundesKaRTELLAMT, Stellungnahme des Bundeskartellamtes zum Entwurf eines Gesetzes zur Weiterentwicklung der Organisationsstrukturen in der Gesetzlichen Krankenversicherung (GKV OrgWG), BT-Drucksache 16/9559 du 16-06-2008; Stellungnahme des Bundeskartellamtes zum Entwurf eines Gesetzes zur Stärkung des Wettbewerbs in der Gesetzlichen Krankenversicherung, BT-Drucksache 16/3100 du 24-10-2006; et Bericht des Bundeskartellamtes über seine Tätigkeit in den Jahren 2005/2006 sowie über die Lage und Entwicklung auf seinem Aufgabengebiet und Stellungnahme der Bundesregierung, BT-Drucksache 16/5710 du 15-06-2007

- BundesRegierung, Wettbewerb im Gesundheitswesen, Positionspapier vom 25. Oktober 2006 (www.die-gesundheitsreform.de)

- Donges J.B., Eekhoff J., Franz W., Möschel W., Neumann M.J.M., Sievert O. (Kronberger KREIS), Mehr Eigenverantwortung und Wettbewerb im Gesundheitswesen, Schriftenreihe der Stiftung Marktwirtschaft, vol. 39, Berlin, 2002

- HAssenteufel P., « Libéralisation ou étatisation de l'assurance maladie ? », Regards sur l'économie allemande, $\mathrm{n}^{\circ} 79 / 2006$

- HeitZer B., « Wettbewerbskontrolle auf den Märkten der gesetzlichen Krankenversicherung », discours tenu le 29-06-2009 à Petersberg (Königswinter), et « Aktuelles aus der Wettbewerbspolitik ordnungspolitisch betrachtet », discours tenu le 01-05-2008 à Kronberg (www.bundeskartellamt.de)

- SACHVERSTÄNDIGENRAT ZUR BEgUTACHTUNG DER GESAMTWIRTSCHAFTLICHEN ENTWICKLUNG, « Gesundheitsreform 2006: Ein misslungener Kompromiss », in Jahresgutachten 2006/07 (\$§ 279-317). 